\title{
Physiological Activation of Synaptic Rac $>$ PAK (p-21 Activated Kinase) Signaling Is Defective in a Mouse Model of Fragile X Syndrome
}

\author{
Lulu Y. Chen, ${ }^{1 *}$ Christopher S. Rex ${ }^{1 *}$ Alex H. Babayan, ${ }^{1}$ Eniko A. Kramár, ${ }^{2}$ Gary Lynch,,${ }^{1,2}$ Christine M. Gall, ${ }^{1,3}$ \\ and Julie C. Lauterborn ${ }^{1}$ \\ Departments of ${ }^{1}$ Anatomy and Neurobiology, ${ }^{2}$ Psychiatry and Human Behavior, and ${ }^{3}$ Neurobiology and Behavior, University of California, Irvine, Irvine, \\ California 92697
}

\begin{abstract}
The abnormal spine morphology found in fragile X syndrome (FXS) is suggestive of an error in the signaling cascades that organize the actin cytoskeleton. We report here that physiological activation of the small GTPase Rac1 and its effector p-21 activated kinase (PAK), two enzymes critically involved in actin management and functional synaptic plasticity, is impaired at hippocampal synapses in the Fmr1knock-out (KO) mouse model of FXS. Theta burst afferent stimulation (TBS) caused a marked increase in the number of synapses associated with phosphorylated PAK in adult hippocampal slices from wild-type, but not Fmr1-KO, mice. Stimulation-induced activation of synaptic Racl was also absent in the mutants. The polymerization of spine actin that occurs immediately after theta stimulation appeared normal in mutant slices but the newly formed polymers did not properly stabilize, as evidenced by a prolonged vulnerability to a toxin (latrunculin) that disrupts dynamic actin filaments. Latrunculin also reversed long-term potentiation when applied at 10 min post-TBS, a time point at which the potentiation effect is resistant to interference in wild-type slices. We propose that a Rac $>$ PAK signaling pathway needed for rapid stabilization of activity-induced actin filaments, and thus for normal spine morphology and lasting synaptic changes, is defective in FXS.
\end{abstract}

\section{Introduction}

Fragile $\mathrm{X}$ mental retardation syndrome arises from an expansion of CGG triplet repeats in the X-linked FMR1 gene resulting in promoter methylation and transcriptional silencing. A potentially critical clue for explaining the cognitive component of FXS came with the discovery that affected individuals have abnormal cortical dendritic spines (Rudelli et al., 1985; Wisniewski et al., 1991; Irwin et al., 2001). Importantly, knocking out fragile $X$ mental retardation protein (FMRP), the FMR1 gene product, in mice produces qualitatively similar disturbances to spine morphology (Comery et al., 1997) as well as impairments in long-term potentiation (LTP) (Larson et al., 2005; Zhao et al., 2005; Lauterborn et al., 2007; Hu et al., 2008). These observations suggest that the fragile-X mutation in some way disturbs cytoskeletal machinery responsible for the anatomy and plasticity of spines, effects that could affect both baseline synaptic transmission and how it is adjusted by learning.

FMRP regulates translation and genetic studies have identified mRNA targets for the protein that are plausibly related to

\footnotetext{
Received Feb. 25, 2010; revised April 19, 2010; accepted May 20, 2010.

Work was supported by National Institutes of Mental Health (NIMH) Grant MH082042 to C.G. and J.L., National Institute of Neurological Disorders and Stroke (NINDS) Grant NS45260 to C.G. and G.L., FRAXA Research Foundation Award FRF-43886 to J.L., NIMH Fellowship MH083396 to L.C., and NINDS Fellowship NS045540 to C.R. We thank Yue Qin Yao for expert technical assistance.

*L. Y. Chen and C. S. Rex contributed equally to this work.

Correspondence should be addressed to Dr. Christine M. Gall, Department of Anatomy and Neurobiology, University of California, Irvine, Irvine CA 92697. E-mail: cmgall@uci.edu.

DOI:10.1523/JNEUROSCI.1077-10.2010

Copyright $\odot 2010$ the authors $\quad 0270-6474 / 10 / 3010977-08 \$ 15.00 / 0$
}

spine cytoskeletal abnormalities (Bardoni and Mandel, 2002; Reeve et al., 2005). The Drosophila FMRP homolog is linked to Rac1, a small GTPase that regulates effectors (e.g., PAK, WASP) important to spine morphology in immature neurons (Billuart and Chelly, 2003; Castets et al., 2005). This is of particular interest because a dominant-negative construct that reduces PAK activity is reported to reverse neocortical spine (and other) abnormalities in Fmr1-knock-outs (KOs) (Hayashi et al., 2004; Hayashi et al., 2007). FMRP has also been implicated in expression of a phosphatase that controls the activity of cofilin (Castets et al., 2005), a protein that regulates the assembly of actin filaments (Bernstein and Bamburg, 2010) as well as spine development. Despite these points, results from initial attempts to identify defects in actin signaling and dynamics in adult Fmr1-KO hippocampus were negative. Theta burst afferent stimulation (TBS), a naturalistic activity pattern commonly used to induce LTP, caused rapid cofilin phosphorylation and actin polymerization at synapses to approximately the same degree in slices from Fmr1-KO and wildtype (WT) mice (Lauterborn et al., 2007).

It seems, then, that the primary spine cytoskeletal problem in FXS involves aspects of actin management beyond the complex processes leading to filament assembly. Actin filament stabilization is one possibility. Newly formed polymers typically enter a dynamic state ("treadmilling") in which they simultaneously add and subtract monomers from opposing ends of the filament, and remain in this condition until disassembled or stabilized (Carlier, 1998; Pollard and Cooper, 2009). Studies using latrunculin, which disrupts treadmilling by blocking actin monomer incor- 
poration, suggest that (1) actin filaments in adult spines are dynamic for several minutes following their formation (Krucker et al., 2000; Rex et al., 2009), and (2) the Rac > PAK pathway promotes filament stabilization (Rex et al., 2009). Prompted by these observations, the present studies investigated the possibility that the PAK-related stabilization of TBS-induced spine actin filaments is impaired in Fmrl-KOs. The results point to a specific hypothesis regarding the causes of spine and synaptic plasticity abnormalities in FXS.

\section{Materials and Methods}

Electrophysiology. Adult (2-3 months) male Fmr1-KO and WT mice (FVB background) were used (Irwin et al., 2002,; Lauterborn et al., 2007). Hippocampal LTP was performed as previously described (Lauterborn et al., 2007). Briefly, transverse hippocampal slices $(300 \mu \mathrm{m})$ were prepared in ice-cold artificial CSF (ACSF) (in mM: $124 \mathrm{NaCl}, 3 \mathrm{KCl}, 1.25 \mathrm{KH}_{2} \mathrm{PO}_{4}$, $3.4 \mathrm{CaCl}_{2}, 2.5 \mathrm{MgSO}_{4}, 26 \mathrm{NaHCO}_{3}$, and 10 dextrose, $\mathrm{pH}$ 7.35). Slices from both genotypes were run simultaneously. Slices were maintained at $31 \pm 1{ }^{\circ} \mathrm{C}$ with surface exposed to humidified $95 \% \mathrm{O}_{2} / 5 \% \mathrm{CO}_{2}$ and ACSF perfused at a rate of $60-70 \mathrm{ml} / \mathrm{h}$. Field EPSPs (fEPSPs) were recorded from the apical dendrites of CA1b pyramidal cells using a glass electrode $(2 \mathrm{M} \mathrm{NaCl})$. Bipolar stimulation was delivered to the apical Schaffer collateral-commissural projections in CAla and CAlc using alternating pulses at $0.05 \mathrm{~Hz}$ with a current that elicited $50 \%$ of the maximal fEPSP response. In experimental slices, synaptic potentiation was induced with a train of 10 theta bursts (i.e., 10 bursts of 4 pulses at $100 \mathrm{~Hz}$, with an interburst interval of $200 \mathrm{~ms}$ ). Yoked control slices from the same mice received low-frequency (3/min) stimulation. Evoked responses were recorded and analyzed for amplitude and falling phase slope. Adenosine was applied by local infusion ( 4 min duration) to field CA1 as described previously (Rex et al., 2009). Theta pulse stimulation ( $3 \mathrm{~min}, 5 \mathrm{~Hz}$ ) was applied at a specified time. The actin polymerization inhibitor latrunculin A (0.2 $\mu \mathrm{M}$; Invitrogen) or vehicle was bath infused via a second perfusion line. For statistical analyses, each slice was considered an "n." Values in text and figures show group means \pm SEM. Statistical significance was assessed using either two-way repeated-measures ANOVA or Mann-Whitney $U$ test.

In situ labeling of filamentous actin. Labeling and quantification of filamentous actin (F-actin) were done as previously described (Lauterborn et al., 2007). Briefly, 45 min after TBS, Alexa 568-phalloidin ( $6 \mu \mathrm{M}$, $4 \mu \mathrm{l}$; Invitrogen) was topically applied 4 times separated by $3 \mathrm{~min}$. Tissue was fixed in $4 \%$ paraformaldehyde, sectioned at $20 \mu \mathrm{m}$ and examined using epifluorescence illumination on a Leica DM6000 microscope. Using in-house software, labeled spine-like structures were counted in high-resolution $Z$-stacks of digital photomicrographs $(0.2 \mu \mathrm{m}$ focal steps; $3 \mu \mathrm{m}$ thick) of proximal stratum (str.) radiatum taken between the two stimulating electrodes (Rex et al., 2007). Counts from three serial sections were averaged to produce a representative value per slice. Each slice was considered an "n." Values in text and figures show group means \pm SEM per $550 \mu \mathrm{m}^{2}$.

Immunohistochemical analyses. Hippocampal slices that received TBS, together with paired control slices from the same mice, were collected at specified poststimulation time points to evaluate dendritic spine levels of GTP-bound (activated) Rac1 or phosphorylated (p) PAK (Ser141), respectively. Specifically, double-immunolabeling for pPAK and the postsynaptic scaffold protein PSD95 or for Rac1-GTP and cofilin was performed (Chen et al., 2007). Cofilin was used as a spine marker in combination with localization of Racl-GTP because the antisera are raised in different species and our work has shown that cofilin is highly localized within hippocampal dendritic spines (Chen et al., 2007). For experiments evaluating basal levels of PAK, adult mouse brains were fast-frozen in 2-methyl butane $\left(-45^{\circ} \mathrm{C}\right)$ and cryostat sectioned on the coronal plane at $20 \mu \mathrm{m}$. The slide-mounted tissue was fixed in $-20^{\circ} \mathrm{C}$ methanol for $15 \mathrm{~min}$ and processed for dual immunohistochemical localization of PAK3 and PSD95. Primary antisera used included mouse anti-PSD95 (1:1000; \#1-054 Affinity BioReagents/Thermo Fisher Scientific), rabbit anti-cofilin (1:250; \#ACFL02, Cytoskeleton), mouse anti-Rac1-GTP (1:1000, \#26903, NewEast Biosciences), rabbit
anti-phospho-PAK1,2,3 Ser141 (1:100; \#44-940G, Invitrogen), and rabbit anti-PAK3 (1:500; \#06-902, Millipore). Alexa 488 anti-mouse IgG and Alexa 594 anti-rabbit IgG (Invitrogen) were used for visualization.

In all cases a sample field of $136 \times 105 \times 3 \mu \mathrm{m}\left(42,840 \mu \mathrm{m}^{3}\right)$ was photographed with a $63 \times$ objective ( 1.4 numerical aperture) and a CCD camera (Orca ER; Hamamatsu Photonics). For LTP experiments, the sample field was placed between the two stimulating electrodes. For analysis of whole brain, sections through mid-septotemporal hippocampus were similarly evaluated. In all instances, digital $Z$-stacks $(0.2 \mu \mathrm{m}$ steps; 3 $\mu \mathrm{m}$ thick) were collected and processed for iterative deconvolution (Volocity 4.1 Restorative Deconvolution, Improvision). Automated inhouse systems were then used to count single- or double-labeled puncta within the size range of dendritic spines. Three-dimensional (3D) analyses of spine immunofluorescent labeling in field CA1 str. radiatum were performed using a multiple intensity threshold series protocol as described previously (Rex et al., 2009; Chen et al., 2010; see supplemental Fig. 1, available at www.jneurosci.org as supplemental material). Briefly, image $Z$-planes were normalized to a target background intensity $(30 \%$ of maximum) and iteratively binarized at regular intensity thresholds (4\% steps ranging from 39 to $78 \%$ of maximum) using exclusion criteria for object size and ellipticity, followed by dilation and erosion filtering. Repeated observations were binned and analyzed to assess object boundaries and discriminate neighboring objects. This process accurately identifies both faintly and densely labeled elements. Finally, elements were reconstructed in 3D to calculate label volume and position. Multiple labels in the same image $Z$-stack were analyzed independently; immunolabeling for the two antigens (spine marker and the target protein) were considered colocalized if any overlap was detected between their respective boundaries. Counts of single-labeled and double-labeled elements from each section were then averaged to obtain a value for each slice or brain. Values in text and figures are group means \pm SEM. Significance was determined by ANOVA and individual comparisons by Tukey's HSD post hoc test.

Western blot analysis. Samples were homogenized in RIPA buffer containing Phosphatase Inhibitor Cocktails 1 and 2 (Sigma-Aldrich) and Complete Protease Inhibitor Cocktail (Roche Diagnostics), separated by $12 \%$ PAGE, and processed for Western blotting using anti-PAK1 (\#2602, Cell Signaling Technology, 1:500), anti-PAK3 (1:500; \#06-902, Millipore), and anti- $\beta$-actin (A1978, Sigma-Aldrich) as described previously (Rex et al., 2007). Immunoreactive bands were measured by film densitometry using ImageJ (National Institutes of Health). Significance was determined by Student's $t$ test.

\section{Results \\ Spine actin management is defective in the Fmr1-KO hippocampus}

Past studies using rhodamine-tagged phalloidin to label filamentous actin showed that TBS elicits significant increases in F-actin in a subpopulation of spines located within the dendritic lamina containing the stimulated contacts (Lin et al., 2005; Kramár et al., 2006; Rex et al., 2007). Tests for the stability of these new actin filaments used latrunculin A, a toxin that prevents the addition of actin monomers and therefore leads to the disassembly of dynamic ("treadmilling”) filaments (Coué et al., 1987). Latrunculin applied to adult hippocampal slices at concentrations that have no effect on baseline synaptic responses, eliminates the increase in densely phalloidinlabeled spines produced by TBS but only when administered shortly after the initial expression of LTP (Rex et al., 2007, 2009); latrunculin infusions started 10 or more minutes after TBS have little if any effect on LTP or phalloidin labeling. These findings indicate that in WT tissue stimulation-induced actin filaments rapidly $(\sim 10 \mathrm{~min})$ transition into a stable state in which they are no longer dependent upon the continuous addition of actin monomers. We tested whether this stabilization process occurs normally in slices prepared from the Fmr1-KO mouse hippocampus.

Latrunculin A or vehicle was applied to field CA1b str. radiatum 10 min after a single TBS train was delivered to that zone's 

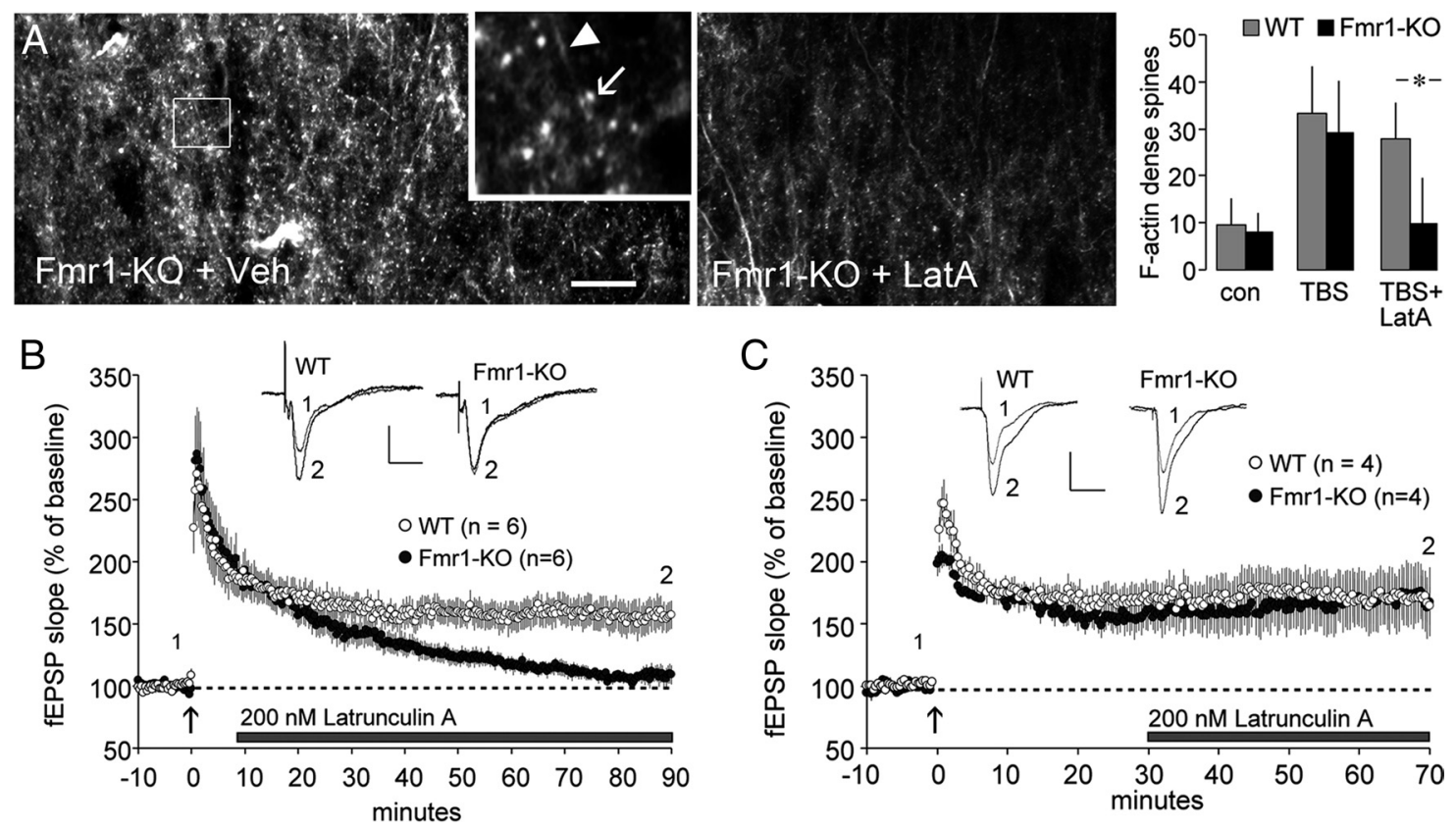

Figure 1. The stabilization of spine F-actin and LTP is impaired in Fmr1-KO hippocampus. A, Photomicrographs (left panels) show in situ Alexa 568-phalloidin labeling of F-actin in spine-like structures in field CA1 of hippocampal slices from K0 mice that received TBS and then vehicle (+Veh) or latrunculin A (+ LatA) treatment 10 min later; slices were harvested 60 min after TBS. Note the abundance of phalloidin labeled F-actin puncta in the +Veh slice and the absence of this labeling in the slice treated with LatA. Inset shows, at higher magnification, the labeling in the field indicated by the box; as shown, dense F-actin labeling is seen in spines (arrow) often in association with lightly labeled dendritic shafts (arrowhead). Scale bar: A, $20 \mu \mathrm{m}$; (for inset), $4 \mu \mathrm{m}$. Bar graph (at right) shows quantification of F-actin-enriched spines in KO and WT slices following control stimulation (con) or TBS with or without LatA infusion. Note: LatA applied at 10 min after TBS significantly reduced F-actin labeling in Fmr1-K0 slices only ( ${ }^{*} p<0.05$; Tukey's HSD post hoc). B, Plot showing fEPSP slopes recorded from CA1 str. radiatum in Fmr1-K0 and WT slices receiving TBS (arrow) and LatA infusion 10 min later. LatA had no effect on LTP in WT slices but abolished it in K0 slices. C, LatA infused at 30 min post-TBS had no effect on potentiation in slices from either genotype. Insets show representative traces (overlaid) collected during baseline (1) or 70-90 min after TBS (2) for each genotype in $\boldsymbol{B}$ and $C$. Calibration: $1 \mathrm{mV}, 10 \mathrm{~ms}$.

Schaffer-commissural afferents. Alexa 568-phalloidin was applied $35 \mathrm{~min}$ later ( $45 \mathrm{~min}$ post-TBS) after which the slices were harvested for microscopic analysis. Digital image $Z$-stacks were collected from the CA1 area of the recording site and phalloidin-labeled elements within the size parameters of dendritic spines were counted using an automated system (Rex et al., 2007). TBS increased the numbers of phalloidin-labeled spines (relative to counts from slices that received control, $3 / \mathrm{min}$ stimulation) to an equivalent extent in vehicletreated slices from WT and Fmrl-KO mice (33.3 \pm 9.9 vs $29.2 \pm$ 11.1 spines per $550 \mu \mathrm{m}^{2}$, for $\mathrm{WT}$ and $\mathrm{KO}$, respectively; $p>0.4$, ANOVA) (Fig. $1 \mathrm{~A}$ ). A quite different result was obtained when latrunculin A was added at $10 \mathrm{~min}$ post-TBS. The treatment did not affect the stimulation-induced increase in labeled spines in WT slices (27.8 \pm 7.7$)$ but eliminated it in slices from KOs $(9.8 \pm 9.9 ; p<0.05$ vs TBS with vehicle alone) (Fig $1 A$ ). These findings constitute the first evidence that spine actin management, and in particular stabilization of newly formed actin filaments, is abnormal in the mouse model of FXS.

Results for LTP paralleled those for F-actin labeling. TBS produced an immediate increase in Schaffer-commissural fEPSP slopes relative to baseline responses in slices from both groups. The magnitude of this early increase was not reliably different between genotypes: $252 \pm 30 \%$ and $265 \pm 29 \%$ at $1-2$ min after TBS for WT and KO slices, respectively. Bath infusion of latrunculin A (200 nM) beginning $10 \mathrm{~min}$ after TBS had no evident effect on LTP in WT slices (156 $\pm 13 \%$ of baseline at $80-90 \mathrm{~min}$ after TBS), but caused a gradual decay in the Fmr1-KO group (Fig. $1 B$ ) such that at $45 \mathrm{~min}$ the fEPSP slope was $108 \pm 5 \%$ of baseline ( $p<0.01$ vs WT). In contrast, latrunculin A infusion at 30 min after TBS had no effect on potentiation in KO slices (Fig. $1 C$ ), indicating that LTP stabilization is achieved in the mutants but at an abnormally slow pace.

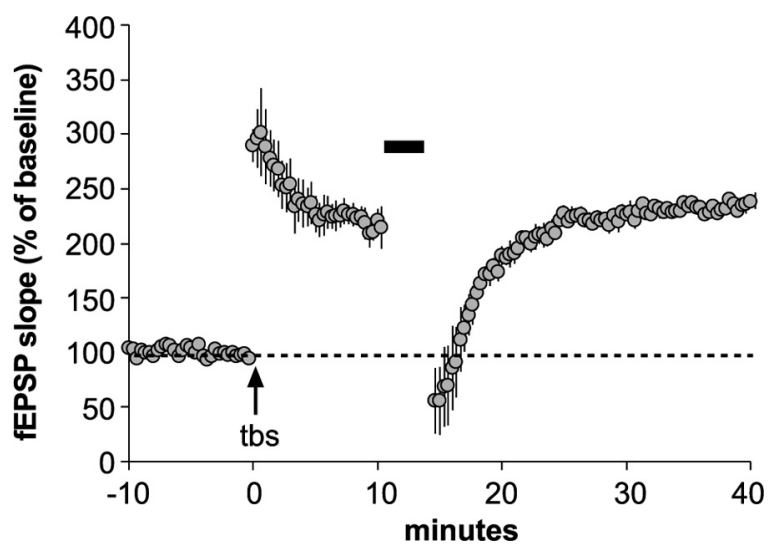

Figure 2. Time period for LTP reversal by manipulations acting on adenosine $A 1$ receptors is not prolonged in Fmr1-K0 mice. Local applications of adenosine (200 $\mu \mathrm{m} ; 4 \mathrm{~min})$ or a 3 min train of $5 \mathrm{~Hz}$ stimulation (bar), two treatments known to reverse LTP when applied immediately after induction (TBS, arrow), had no lasting effects on potentiation when applied at $10 \mathrm{~min}$ after TBS in hippocampal slices ( $n=4)$ prepared from Fmr1-KO mice. Both manipulations caused a transient depression of synaptic responses, as expected for stimulation of adenosine A1 receptors.

Finally, we ran four additional slices to test whether reversal of LTP via time-dependent manipulations that disrupt the assembly, as opposed to the stabilization, of actin filaments were also effective over a prolonged post-TBS time frame in KO hippocampus. Comparable results were obtained with $3 \mathrm{~min}$ of $5 \mathrm{~Hz}$ afferent stimulation or local application of adenosine $(200 \mu \mathrm{M})$, manipulations known to reverse LTP in an adenosine A1 receptor-dependent manner (Larson et al., 1993); the combined results are presented in Figure 2. As shown, application of the 
treatments at 10 min after TBS had no detectable effect on LTP in Fmr1-KO slices. These results further illustrate the selectivity of the impairments produced by the loss of the FMRP.

\section{TBS fails to activate PAK at Fmr1-KO synapses}

Recent results led to the proposal that TBS activates two Rho GTPase signaling cascades that differentially contribute to the rapid cytoskeletal reorganization in adult spines and the generation of stable LTP (Rex et al., 2009). The first of these (a RhoA $>$ ROCK $>$ LIM-K $>$ cofilin sequence) triggers actin polymerization whereas the second $(\mathrm{Rac}>\mathrm{PAK})$ appears to stabilize the new actin filaments. This argument makes PAK, which has been implicated in FXS by other lines of evidence (Boda et al., 2004; Hayashi et al., 2007), a plausible contributor to the stabilization defects described above. We therefore tested whether TBS causes phosphorylation (activation) of synaptic PAK in Fmr1-KO slices. A single train of 10 theta bursts, or control stimulation, was delivered to two populations of Schaffer-commissural afferents converging on proximal CAlb str. radiatum and the slices were collected 7 min later. The tissue was processed for immunofluorescence double-labeling for the postsynaptic density protein PSD95 and pPAK (Ser141 of PAKs 1,2,3), and then evaluated using a multi-intensity threshold series protocol for the incidence of single- and double-labeled puncta (supplemental Fig. 1, available at www. jneurosci.org as supplemental material; also see supplemental Video 1).

Dense concentrations of pPAK were localized to only a small percentage of the synaptic population in field CA1 of Fmr1-KO $(4.2 \pm 1.9 \%)$ and WT $(3.8 \pm 0.8 \%)$ slices that received control, low-frequency stimulation (Fig. 3A). These observations accord with previous evidence for low levels of actin signaling at the great majority of synapses in adult hippocampus (Chen et al., 2007). There were no detectable differences $(<10 \% ; p>0.50)$ between genotypes with regard to numbers of pPAK puncta that were, or were not, colocalized with PSD95. We conclude from these data that the mutation does not block constitutive activation of PAK at spine synapses.

Very different results were obtained for TBS-driven activation of PAK. As expected from past studies (Chen et al., 2007; Rex et al., 2009), TBS caused a large increase $(85 \pm 31 \%)$ in the number of PSD95-immunopositive $(+)$ synapses associated with dense concentrations of pPAK (pPAK + PSDs) in WT slices at $7 \mathrm{~min}$ after TBS ( $p=0.014$, post hoc test following ANOVA). This increase was transient: numbers of PPAK+ PSDs were comparable to control values at 15 and 30 min post-TBS (Fig. $3 C, D$ ). In contrast to the WTs, TBS failed to increase the number of pPAK+ PSDs at 7, 15 or 30 min post-TBS in Fmr1-KO slices $(-11 \pm$ $26 \%$; $p>0.30$ for $7 \mathrm{~min}$ vs yoked controls) (Fig. 3D). These
A
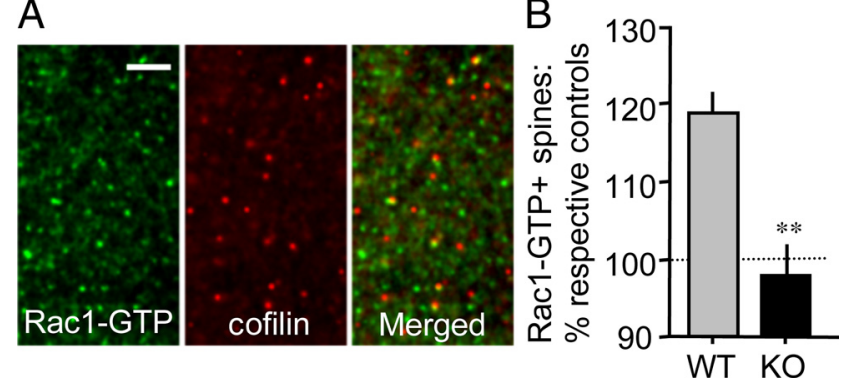

Figure 4. TBS fails to activate Rac1 in Fmr1-KO hippocampal spines. $\boldsymbol{A}$, Photomicrographs show immunoreactivity for Rac1-GTP (green) and cofilin (red; spine marker), and merged image, in CA1 str. radiatum of a WT, control hippocampal slice. As shown, activated Rac1 is localized to a subpopulation of cofilin-labeled spines. Scale bar, $5 \mu \mathrm{m}$. $\boldsymbol{B}$, Quantitative analysis shows the effect of TBS on Rac1-GTP ${ }^{+}$spines in the two genotypes (levels normalized to respective genotype low-frequency stimulation controls; $n=$ 9-11 slices/group). As shown, TBS increased the number of Rac1-GTP ${ }^{+}$spines in slices from WT but not Fmr1-K0 mice. ${ }^{* *} p<0.001$, one-tailed Student's $t$ test; planned comparison. 
A

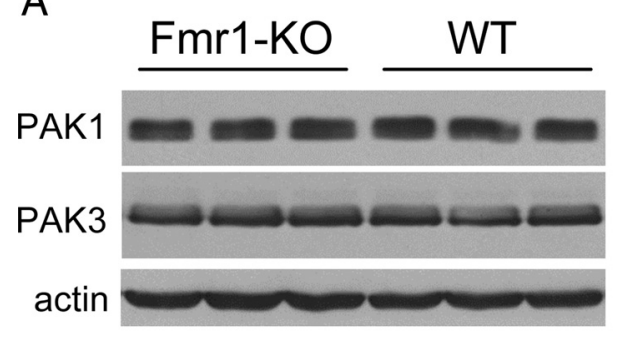

B

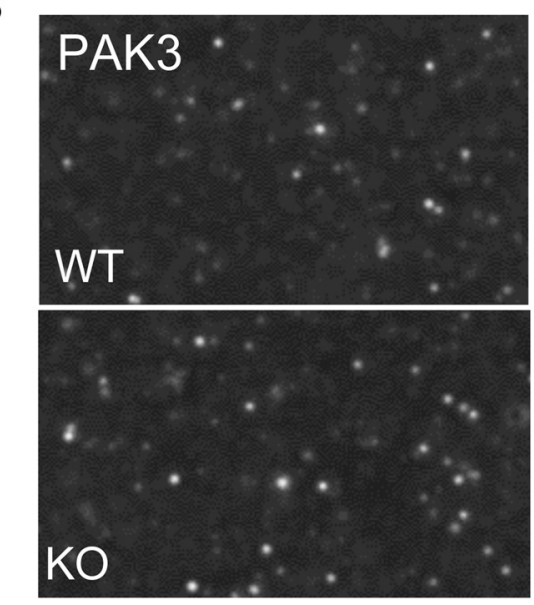

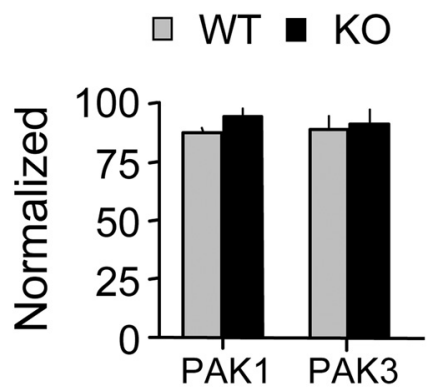

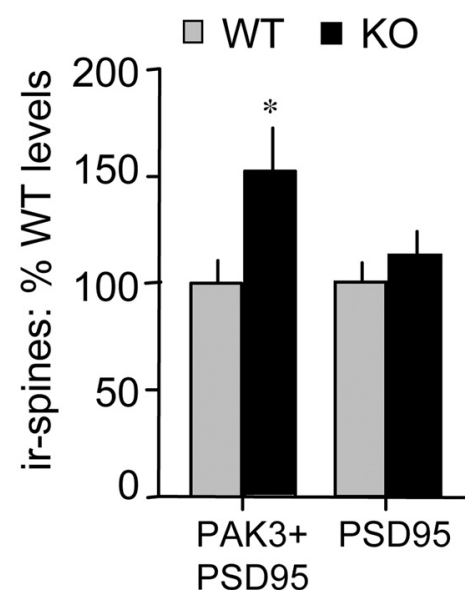

Figure 5. Abnormal PAK3 levels in Fmr1-K0 spines. $\boldsymbol{A}$, Left, Western blots show total levels of PAK1, PAK3, and actin in hippocampal homogenates from Fmr1-KO and WT mice. Right, Quantitative analysis shows no difference in hippocampal PAK levels (immunoreactive band ODs) between genotypes; levels normalized to $\beta$-actin ( $n=4 /$ group). $\boldsymbol{B}$, Left, Photomicrographs show PAK3 immunolabeling of spine-like puncta in CA1 str. radiatum of WT and Fmr1-K0 mice. Right, Bar graph shows quantification of PAK3 ${ }^{+}$spines (i.e., PAK3 ${ }^{+}$PSD95 double-labeled puncta) and total numbers of PSD95 ${ }^{+}$spines in the CA1 str. radiatum sample field from Fmr1-K0 and WT mice ( $n=5-8$ mice/group). Results are expressed as percentage of mean WT control values; ${ }^{*} p<0.05$, Tukey's HSD post hoc, KO vs WT group.

results confirm the experimental prediction that activation of PAK by afferent stimulation is defective at Fmr1-KO synapses relative to the effects seen in WTs ( $p=0.035)$.

\section{Factors contributing to the failure of PAK activation in Fmr1-KO mice}

The most straightforward explanation for the loss of PAK phosphorylation in response to TBS is a defect in physiological activation of the kinase's upstream regulators Rac/Cdc42. We tested this using immunolabeling with one antibody for activated Rac (i.e., Rac1-GTP) and a second directed at cofilin, a spine marker (Racz and Weinberg, 2006; Chen et al., 2007) (Fig. 4A). Hippocampal slices were harvested $2 \mathrm{~min}$ after receiving 10-burst TBS or control stimulation and processed for double-labeling immunofluorescence. High levels of Racl-GTP labeling were found in $19 \pm 1 \%$ of the cofilin-dense puncta in field CA1 of control-stimulated WT slices, a substantially greater degree of double-labeling than observed for pPAK in spines. Total values for cofilin + puncta in control WT and Fmrl-KO slices were within $4 \%$ of each other as were the mean numbers for doublelabeled profiles. It thus appears that baseline activity of the Rac $>$ PAK cascade within spines is not significantly affected by the loss of FMRP.

In WT slices, TBS produced a significant $(18 \pm 3 \%)$ increase in the number of spines containing activated Racl relative to measures from control slices that received low-frequency stimulation $(p=0.003)$. Given the high levels of baseline double- labeling, this increase involves $3 \%$ to $4 \%$ of the total population of cofilin + structures, a value similar to that estimated for the increase in $\mathrm{pPAK}+$ synapses with TBS. In contrast, there was no effect of TBS on the numbers of spines containing activated Racl in slices from Fmr1-KO mice $(1 \pm 5 \%$ above yoked control Fmr1-KO slices; $p>0.4$ ) (Fig. $4 B$ ). The difference in the effect of TBS in the two genotypes was highly significant $(p=$ 0.004 for WT vs Fmrl-KO). These data confirm that a principle upstream activator of PAK fails to respond to LTPinducing stimulation in the Fmrl-KO hippocampus.

Changes in PAK concentrations within a given cellular compartment could have pronounced functional consequences because of the complexity of events required to fully activate the enzyme. As described, the incidence of pPAK at Fmr1-KO synapses did not differ from WT values, but this result does not rule out the possibility that concentrations of total (phosphorylated and unphosphorylated) PAK1 or PAK3 are affected by the mutation. We first tested this point using Western blots and found that basal concentrations of both PAK1 and PAK3 are comparable in WT and Fmrl-KO hippocampus (Fig. $5 A$ ). Because FMRP associates with various transport proteins (Ohashi et al., 2002) and its loss could therefore alter the distribution of PAK within neurons, we next assessed levels of PAK immunoreactivity at spine synapses using double immunostaining for PSD95 and total PAK3. This analysis indicated that the number of synapses associated with dense concentrations of PAK3 is $\sim 50 \%$ greater in field CA1 str. radiatum in the mutants relative to WT mice $(p<0.05)$. The total number of PSD95+ contacts was comparable for the two groups (Fig. 5B). Together, the Western blot and immunostaining results suggest that the fragile X mutation leads to an accumulation of PAK in dendritic spines, an effect that could interact with the loss of Rac1 responsivity to afferent stimulation to impair activation of synaptic PAK.

\section{Discussion}

At least two actin signaling cascades responsible for morphological transformations across cell types are engaged by TBS, in an NMDA receptor-dependent fashion, at adult brain synapses (Chen et al., 2007; Rex et al., 2009). The present findings indicate that the loss of FMRP eliminates the effects of synaptic activity on one of these pathways and does so with surprising selectivity. As discussed below, there are good reasons to conclude that this defect is directly involved in two of the essential elements of the FXS phenotype: aberrant spine anatomy and learning disabilities.

The first of the actin regulatory pathways set in motion by TBS involves RhoA, its effector RhoA kinase (ROCK), and the actin severing protein cofilin. The latter disrupts actin filament formation, and cofilin inactivation (via phosphorylation) is essential for actin polymerization in many cell types and experimental circumstances (Bernstein and Bamburg, 2010). Agents that in- 
hibit RhoA or ROCK block TBS-induced cofilin phosphorylation at synapses along with the increases in spine F-actin that accompany phosphorylation (Rex et al., 2009). Previously, we showed that both theta-driven effects are normal in Fmr1KOs (Lauterborn et al., 2007), indicating that the mutation leaves intact events leading from transient NMDA receptor stimulation through RhoA activation to actin filament assembly.

TBS also causes phosphorylation (activation) of PAK, a pivotal enzyme in actin management (Bokoch, 2003), at WT synapses. PAK activity is largely controlled by the Rho GTPases Rac and Cdc42 and, in accord with this, specific Rac inhibitors disrupt PAK's response to TBS (Rex et al., 2009). We report here that theta-driven PAK phosphorylation is missing at excitatory synapses in Fmr1-KO hippocampal slices. This constitutes the first direct evidence that physiological activation of a primary actin regulator in adult dendritic spines is impaired by the FXS mutation. Although inhibitors of Rac or PAK have no clear effect on baseline synaptic transmission or the initial expression of LTP in rat hippocampal slices, both block the stabilization of F-actin assembled in the 30$120 \mathrm{~s}$ following TBS (Rex et al., 2009; our unpublished observations). These results, and related evidence, led to the hypothesis that activation of the RhoA-to-cofilin cascade generates actin polymers that remain in an unstable state until acted upon by another sequence of events including upstream Rac $>$ PAK signaling. This idea is consistent with evidence that PAK regulates actin cytoskeletal architecture and stability through various enzymes and adaptor molecules (Szczepanowska, 2009), including cortactin (Webb et al., 2006), a protein that interacts with the Arp2/3 complex to promote filament branching and stabilization (Weaver et al., 2003). It also accords with evidence that LTP is impaired in mice lacking Arp2/3 regulating enzyme WAVE-1 (Soderling et al., 2007).

The clear prediction from the above arguments is that the absence of a PAK response to TBS at Fmr1-KO synapses will be accompanied by a failure to stabilize activity-induced actin filaments and thus changes in cytoskeletal branching underlying spine morphology. The present studies confirm that the F-actin stabilization process is defective in Fmr1-KOs: latrunculin A, which disrupts dynamic actin filaments, eliminated newly polymerized actin when applied at $10 \mathrm{~min}$ post-TBS, a time point at which it has no effect in rats or WT mice (Rex et al., 2009; present results). In all, it appears that a selective impairment to the Rac $>$ PAK pathway leaves the Fmr1-KO system competent for initial activity-induced reorganization of the spine actin cytoskeleton but interferes with its capacity for maintaining those changes.

A significant body of evidence indicates that LTP consolidation relies on stabilization of the spine actin cytoskeleton (Smart et al., 2003; Dillon and Goda, 2005; Bramham, 2008; Lynch et al., 2008; Rex et al., 2009; Kasai et al., 2010). Although the present results demonstrate that the latter process is impaired in mutants, we also found latrunculin had no effect on F-actin and potentiation in the KOs when infusions were started at 30 min after TBS. Thus, new actin filaments do eventually stabilize but at a slower rate than found in WTs. Time course analyses showed that the eventual stabilization of F-actin is not due to a delayed activation of PAK. This implies that while Rac $>$ PAK signaling is important for the normal, WT pace of consolidation, its absence in the mutants can be compensated for by slower, as yet unknown, mechanisms. An interesting possibility is that signaling through the Rho GTPase Cdc42, which regulates Arp2/3-mediated F-actin nucleation and branching independent of PAK (Stradal et al., 2004), may effect stabilization in the mutants.

Why PAK fails to respond to TBS is an open question. We demonstrate here that physiological activation of synaptic Rac is absent in Fmr1-KO slices, likely causing, or contributing to, the failed down-stream phosphorylation of PAK. However, baseline numbers of synapses with high levels of Rac1-GTP, and pPAK, 
were comparable in mutants and WTs; this indicates that at least some activation pathways (Leisner et al., 2005; Bokoch, 2003) are still functional in Fmr1-KO hippocampus. The presence of seemingly normal F-actin assembly and induction of LTP by TBS further constrains possible explanations for the impaired Rac $>$ PAK signaling because these effects depend upon proper functioning of a complex array of receptors and intracellular signaling molecules (Lynch et al., 2008; Kramár et al., 2009). An essential defect is thus likely to lie in signaling through guanine regulatory factors with some selectivity for Rac or in pathways linking the LTP induction machinery to those factors (Fig. 6).

Abnormal PAK distributions could also interfere with its activation in the mutants. PAK3 immunoreactivity was elevated in KO dendritic spines although total PAK levels, in hippocampal homogenates, were comparable in KOs and WTs. Activation of PAK requires multiple kinases (Chong et al., 2001) and therefore is likely to involve third order kinetics. Excess levels of spine PAK3 could thus reduce phosphorylation at the kinase's activation site by presenting an unusually high number of target protein copies (PAK) relative to drivers (e.g., Rac1, Cdc42) within this particular cellular compartment.

With regard to mechanisms that could account for the unusual spine concentrations of PAK3, it is noteworthy that FMRP interacts with several motor proteins including myosin $\mathrm{Va}$ (Ohashi et al., 2002), which is thought to be involved in the translocation of materials into spine heads (Petralia et al., 2001). Moreover, myosin light chain kinase is a target of PAK signaling. Thus, the abnormal spine concentrations of PAK described here could arise from impairments in myosin motor function downstream from Rac $>$ PAK signaling.

Previous work has implicated PAK in synaptic plasticity and cognitive impairments (Boda et al., 2004; Meng et al., 2005), including in FXS. Hayashi et al. (2007) found that forebrain expression of a dominant-negative PAK transgene restored stabilization of neocortical LTP and partially normalized behavioral measures in Fmr1-KO mice. Although effects in hippocampus, and spine levels of active or total PAK, were not measured in these studies, the results suggest that reducing PAK activity restores some measures of synaptic plasticity in Fmr1-KO mice. Work with Drosophila has shown that impairments to long term memory arise from increasing or decreasing expression of the homolog of filamin A, a PAK target involved in F-actin stabilization (Bolduc et al., 2010). Together with the present results, these findings point to the conclusion that abnormal (elevated or depressed) PAK signaling to actin disturbs the stabilization of spine F-actin, LTP and memory.

The consolidation deficit described here is suggestive of a learning disability in which disrupting stimuli play a prominent role. Brain activity patterns that are commonplace during behavior can reverse recently formed LTP (Colgin et al., 2004). Presumably, then, any slowing of LTP consolidation would increase the likelihood that information encoded by synaptic potentiation will be degraded by these routine "distractions." While speculative, these points make explicit predictions about the temporal ordering of events that will maximize (or minimize) learning deficits in Fmr1-KOs and FXS.

Finally, our results accord with the widely discussed idea that spine cytoskeletal abnormalities are major contributors to an array of neuropsychiatric disorders (Fiala et al., 2002; Lynch et al., 2008; Nadif Kasri and Van Aelst, 2008). Seen in this light, the Fmr1-KO abnormalities could be a special case of a broad phenomenon. It is therefore of interest with regard to possible FXS therapeutics that brain-derived neurotrophic factor (BDNF) promotes PAK phosphorylation (Rex et al., 2007) and rescues functional synaptic plasticity in multiple animal models of cognitive impairment (Rex et al., 2006; Lynch et al., 2007; Kramár et al., 2009); tests in two of these cases show that BDNF also restores activity-induced cytoskeletal remodeling. We previously found that BDNF rescues hippocampal LTP in Fmr1-KOs (Lauterborn et al., 2007) and are currently testing whether it has comparable effects on spine actin signaling and F-actin stabilization.

\section{References}

Bardoni B, Mandel JL (2002) Advances in understanding of fragile X pathogenesis and FMRP function, and in identification of $\mathrm{X}$ linked mental retardation genes. Curr Opin Genet Dev 12:284-293.

Bernstein BW, Bamburg JR (2010) ADF/Cofilin: a functional node in cell biology. Trends Cell Biol 20:187-195.

Billuart P, Chelly J (2003) From fragile X mental retardation protein to Rac1 GTPase: new insights from Fly CYFIP. Neuron 38:843-845.

Boda B, Alberi S, Nikonenko I, Node-Langlois R, Jourdain P, Moosmayer M, Parisi-Jourdain L, Muller D (2004) The mental retardation protein PAK3 contributes to synapse formation and plasticity in hippocampus. J Neurosci 24:10816-10825.

Bokoch GM (2003) Biology of the p21-activated kinases. Annu Rev Biochem 72:743-781.

Bolduc FV, Bell K, Rosenfelt C, Cox H, Tully T (2010) Fragile X mental retardation 1 and filamin A interact genetically in Drosophila long-term memory. Front Neural Circuits 3:22.

Bramham CR (2008) Local protein synthesis, actin dynamics, and LTP consolidation. Curr Opin Neurobiol 18:524-531.

Carlier MF (1998) Control of actin dynamics. Curr Opin Cell Biol 10:45-51.

Castets M, Schaeffer C, Bechara E, Schenck A, Khandjian EW, Luche S, Moine H, Rabilloud T, Mandel JL, Bardoni B (2005) FMRP interferes with the Racl pathway and controls actin cytoskeleton dynamics in murine fibroblasts. Hum Mol Genet 14:835-844.

Chen LY, Rex CS, Casale MS, Gall CM, Lynch G (2007) Changes in synaptic morphology accompany actin signaling during LTP. J Neurosci 27:5363-5372.

Chen LY, Rex CS, Sanaiha Y, Lynch G, Gall CM (2010) Learning induces neurotrophin signaling at hippocampal synapses. Proc Natl Acad Sci U S A 107:7030-7035.

Chong C, Tan L, Lim L, Manser E (2001) The mechanism of PAK activation. Autophosphorylation events in both regulatory and kinase domains control activity. J Biol Chem 276:17347-17353.

Colgin LL, Kubota D, Jia Y, Rex CS, Lynch G (2004) Long-term potentiation is impaired in rat hippocampal slices that produce spontaneous sharp waves. J Physiol 558:953-961.

Comery TA, Harris JB, Willems PJ, Oostra BA, Irwin SA, Weiler IJ, Greenough WT (1997) Abnormal dendritic spines in fragile X knockout mice: maturation and pruning deficits. Proc Natl Acad Sci USA 94:5401-5404.

Coué M, Brenner SL, Spector I, Korn ED (1987) Inhibition of actin polymerization by latrunculin A. FEBS Lett 213:316-318.

Dillon C, Goda Y (2005) The actin cytoskeleton: integrating form and function at the synapse. Annu Rev Neurosci 28:25-55.

Fiala JC, Spacek J, Harris KM (2002) Dendritic spine pathology: cause or consequence of neurological disorders? Brain Res Rev 39:29-54.

Hayashi ML, Choi SY, Rao BS, Jung HY, Lee HK, Zhang D, Chattarji S, Kirkwood A, Tonegawa S (2004) Altered cortical synaptic morphology and impaired memory consolidation in forebrain-specific dominantnegative PAK transgenic mice. Neuron 42:773-787.

Hayashi ML, Rao BS, Seo JS, Choi HS, Dolan BM, Choi SY, Chattarji S, Tonegawa $\mathrm{S}$ (2007) Inhibition of p21-activated kinase rescues symptoms of fragile $\mathrm{X}$ syndrome in mice. Proc Natl Acad Sci U S A 104:11489-11494.

Hu H, Qin Y, Bochorishvili G, Zhu Y, van Aelst L, Zhu JJ (2008) Ras signaling mechanisms underlying impaired GluR1-dependent plasticity associated with fragile X syndrome. J Neurosci 28:7847-7862.

Irwin SA, Patel B, Idupulapati M, Harris JB, Crisostomo RA, Larsen BP, Kooy F, Willems PJ, Cras P, Kozlowski PB, Swain RA, Weiler IJ, Greenough WT (2001) Abnormal dendritic spine characteristics in the temporal and visual cortices of patients with fragile-X syndrome: a quantitative examination. Am J Med Genet 98:161-167.

Irwin SA, Idupulapati M, Gilbert ME, Harris JB, Chakravarti AB, Rogers EJ, 
Crisostomo RA, Larsen BP, Mehta A, Alacantara CJ, Patel B, Swain RA, Weiler IJ, Oostra BA, Greenough WT (2002) Dendritic spine and dendritic field characteristics on layer $\mathrm{V}$ pyramidal neurons in the visual cortex of fragile-X knockout mice. Am J Med Genet 111:140-146.

Kasai H, Fukuda M, Watanabe S, Hayashi-Takagi A, Noguchi J (2010) Structural dynamics of dendritic spines in memory and cognition. Trends Neurosci 33:121-129.

Kramár EA, Lin B, Rex CS, Gall CM, Lynch G (2006) Integrin-driven actin polymerization consolidates long-term potentiation. Proc Natl Acad Sci U S A 103:5579-5584.

Kramár EA, Chen LY, Brandon NJ, Rex CS, Liu F, Gall CM, Lynch G (2009) Cytoskeletal changes underlie estrogen's acute effects on synaptic transmission and plasticity. J Neurosci 29:12982-12993.

Krucker T, Siggins GR, Halpain S (2000) Dynamic actin filaments are required for stable long-term potentiation (LTP) in area CA1 of the hippocampus. Proc Natl Acad Sci U S A 97:6856-6861.

Larson J, Xiao P, Lynch G (1993) Reversal of LTP by theta frequency stimulation. Brain Res 600:97-102.

Larson J, Jessen RE, Kim D, Fine AK, du Hoffmann J (2005) Age-dependent and selective impairment of long-term potentiation in the anterior piriform cortex of mice lacking the fragile $\mathrm{X}$ mental retardation protein. J Neurosci 25:9460-9469.

Lauterborn JC, Rex CS, Kramár E, Chen LY, Pandyarajan V, Lynch G, Gall CM (2007) Brain-derived neurotrophic factor rescues synaptic plasticity in a mouse model of fragile X syndrome. J Neurosci 27:10685-10694.

Leisner TM, Liu M, Jaffer ZM, Chernoff J, Parise LV (2005) Essential role of ClB1 in regulating PAK1 activation and cell migration. J Cell Biol 170:465-476.

Lin B, Kramár EA, Bi X, Brucher FA, Gall CM, Lynch G (2005) Theta stimulation polymerizes actin in dendritic spines of hippocampus. J Neurosci 25:2062-2069.

Lynch G, Kramár EA, Rex CS, Jia Y, Chappas D, Gall CM, Simmons DA (2007) Brain-derived neurotrophic factor restores synaptic plasticity in a knock-in mouse model of Huntington's disease. J Neurosci 27:4424-4434.

Lynch G, Rex CS, Chen LY, Gall CM (2008) The substrates of memory: defects, treatments, and enhancement. Eur J Pharmacol 585:2-13.

Meng J, Meng Y, Hanna A, Janus C, Jia Z (2005) Abnormal long-lasting synaptic plasticity and cognition in mice lacking the mental retardation gene Pak3. J Neurosci 25:6641-6650.

Nadif Kasri N, Van Aelst L (2008) Rho-linked genes and neurological disorders. Pflugers Arch 455:787-797.

Ohashi S, Koike K, Omori A, Ichinose S, Ohara S, Kobayashi S, Sato TA, Anzai $\mathrm{K}$ (2002) Identification of mRNA/protein (mRNP) complexes containing Puralpha, mStaufen, fragile X protein, and myosin Va and their association with rough endoplasmic reticulum equipped with a kinesin motor. J Biol Chem 277:37804-37810.

Petralia RS, Wang YX, Sans N, Worley PF, Hammer JA 3rd, Wenthold RJ
(2001) Glutamate receptor targeting in the postsynaptic spine involves mechanisms that are independent of myosin Va. Eur J Neurosci 13:1722-1732.

Pollard TD, Cooper JA (2009) Actin, a central player in cell shape and movement. Science 326:1208-1212.

Racz B, Weinberg RJ (2006) Spatial organization of cofilin in dendritic spines. Neuroscience 138:447-456.

Reeve SP, Bassetto L, Genova GK, Kleyner Y, Leyssen M, Jackson FR, Hassan BA (2005) The Drosophila fragile X mental retardation protein controls actin dynamics by directly regulating profilin in the brain. Curr Biol 15:1156-1163.

Rex CS, Lauterborn JC, Lin CY, Kramár EA, Rogers GA, Gall CM, Lynch G (2006) Restoration of long-term potentiation in middle-aged hippocampus after induction of brain-derived neurotrophic factor. J Neurophysiol 96:677-685.

Rex CS, Lin CY, Kramár EA, Chen LY, Gall CM, Lynch G (2007) Brainderived neurotrophic factor promotes long-term potentiation-related cytoskeletal changes in adult hippocampus. J Neurosci 27:3017-3029.

Rex CS, Chen LY, Sharma A, Liu J, Babayan AH, Gall CM, Lynch G (2009) Different Rho GTPase-dependent signaling pathways initiate sequential steps in the consolidation of long-term potentiation. J Cell Biol 186:85-97.

Rudelli RD, Brown WT, Wisniewski K, Jenkins EC, Laure-Kamionowska M, Connell F, Wisniewski HM (1985) Adult fragile X Syndrome. Cliniconeuropathologic findings. Acta Neuropathol 67:289-295.

Smart FM, Edelman GM, Vanderklish PW (2003) BDNF induces translocation of initiation factor $4 \mathrm{E}$ to mRNA granules: evidence for a role of synaptic microfilaments and integrins. Proc Natl Acad Sci U S A 100:14403-14408.

Soderling SH, Guire ES, Kaech S, White J, Zhang F, Schutz K, Langeberg LK, Banker G, Raber J, Scott JD (2007) A WAVE-1 and WRP signaling complex regulates spine density, synaptic plasticity, and memory. J Neurosci 27:355-365.

Stradal TE, Rottner K, Disanza A, Confalonieri S, Innocenti M, Scita G (2004) Regulation of actin dynamics by WASP and WAVE family proteins. Trends Cell Biol 14:303-311.

Szczeponowska J (2009) Involvement of Rac/Cdc42/PAK pathway in cytoskeletal rearrangements. Acta Biochim Pol 56:225-234.

Weaver AM, Young ME, Lee WL, Cooper JA (2003) Integration of signals to the Arp2/3 complex. Curr Opin Cell Biol 15:23-30.

Webb BA, Zhou S, Eves R, Shen L, Jia L, Mak AS (2006) Phosphorylation of cortactin by p21-activated kinase. Arch Biochem Biophys 456:183-193.

Wisniewski KE, Segan SM, Miezejeski CM, Sersen EA, Rudelli RD (1991) The $\operatorname{Fra}(\mathrm{X})$ syndrome: neurological, electrophysiological, and neuropathological abnormalities. Am J Med Genet 38:476-480.

Zhao MG, Toyoda H, Ko SW, Ding HK, Wu LJ, Zhuo M (2005) Deficits in trace fear memory and long-term potentiation in a mouse model for fragile X syndrome. J Neurosci 25:7385-7392. 